\title{
Origin of Borromean systems
}

\author{
E. Garrido ${ }^{\mathrm{a}}$, D.V. Fedorov ${ }^{\mathrm{b}}$, A.S. Jensen ${ }^{\mathrm{b}}$ \\ a Instituto de Estructura de la Materia, CSIC, Serrano 123, E-28006 Madrid, Spain \\ ${ }^{\mathrm{b}}$ Department of Physics and Astronomy, University of Aarhus, DK-8000 Aarhus C, Denmark \\ Received 16 March 2004; received in revised form 9 June 2004; accepted 12 June 2004 \\ Available online 15 September 2004 \\ Editor: J.-P. Blaizot
}

\begin{abstract}
The complex energies of the three-body resonances for one infinitely heavy particle and two non-interacting light particles are the sum of the two contributing two-body complex resonance energies. The bound state of a Borromean system originates from a resonance when the third interaction is introduced, a finite mass is allowed and proper angular momentum coupling is included. The relative importance of these contributions are investigated and the resulting structure of Borromean systems are traced back to the two-body continuum properties. The $0^{+}$and $2^{+}$states in ${ }^{6} \mathrm{He}$ result from neutron-core $p$-states and the ground and first excited state of ${ }^{11} \mathrm{Li}$ originate from neutron-core $s^{2}$ and $s p$-states.
\end{abstract}

(C) 2004 Elsevier B.V. All rights reserved.

PACS: 21.45.+v; 31.15.Ja; 25.70.Ef

\section{Introduction}

Three particles can form a bound state even when none of the two-body subsystems are bound [1]. The attraction responsible for such Borromean states is almost inevitably of short range. The existence was highlighted in the measurements of the unexpected large reaction cross section of ${ }^{11} \mathrm{Li}$ implying a very large radius [2]. Subsequently halos were used to denote such systems [3] with simple scaling properties but independent of the interactions [4]. Necessary con-

E-mail address: imteg57@ pinar2.csic.es (E. Garrido). ditions for halo occurrence are small binding energy, low relative angular momentum and a vanishing or relatively weak repulsive Coulomb interaction.

Borromean systems are by definition weakly bound, since the three-body binding eventually originates from two-body interactions which must be too weak to bind the two-body systems. The stability of Borromean systems is surprising because a realistic system of a comparatively heavy core surrounded by two mutually non-interacting particles has three-body resonance energies equal to the sum of two particle-core resonance energies [5]. Thus if none are bound the three-body system is also unbound and the Borromean system does not exist. 
However, three different effects can independently change this conclusion, i.e., a particle-particle attraction, a finite core-mass allowing favorable angular momentum coupling, and the smaller kinetic energy in the three-body system compared to the three two-body systems. The purpose of the present Letter is to estimate these three effects and map out the road from the two-body continuum to Borromean states. We shall concentrate on systems where one particle mass is substantially larger than the other two. Then our initial schematic structure of one infinitely heavy mass and two non-interacting particles still leaves strong fingerprints in the final system with realistic masses and interactions. Systems with comparable masses can also be studied in the same way but now the realistic system can have rather little in common with the initial schematic configuration.

In the quantitative description we shall use universal terms suitable for understanding the generic origin of Borromean systems in general and in particular the well studied nuclear examples of ${ }^{6} \mathrm{He}$ and ${ }^{11} \mathrm{Li}$. A welcome side effect is that possible three-body (continuum and bound-state) structures become easier to predict directly from properties of the two-body interactions.

\section{The foundation}

We label the three particles by $i=1,2,3$ and denote their masses and two-body interactions by $m_{i}$ and $V_{i j}$. All three interactions are too weak to support a bound state. The two-body energies of the virtual states and resonances are $E_{i j}^{(n)}$. For the schematic system, where $V_{23}=0$ and $m_{1}=\infty$, the three-body system has corresponding resonance and virtual state energies $E=E_{12}^{(n)}+E_{13}^{(m)}$. This addition theorem holds for all angular momenta compatible with the usual coupling rules. The energy is degenerate if more than one total angular momentum is allowed. These observations follow from the separability of the two subsystems [5].

The procedure is now to relax the stringent conditions of the schematic model until a realistic Borromean system is reached. It is then convenient to maintain the two-body properties of the two initially interacting two-body systems. This is achieved with unchanged potentials $V_{12}, V_{13}$ and reduced masses $\mu_{12}, \mu_{13}$ (vary $m_{2}$ and $m_{3}$ ) as $m_{1}$ becomes finite. The three-body resonances can then be studied as function of $m_{1}$ for different partial waves, angular momenta, parities, symmetries, and for non-vanishing $V_{23}$. Conditions for the appearance of Borromean systems must then emerge.

For spin independent two-body interactions the three-body wave function factorizes into coordinate and spin parts. The energies of the three-body resonances and bound states are then independent of the particle spins and their couplings. This is still valid when particles 2 and 3 are identical fermions or bosons. The spin part of the wave function can always be used to establish the correct (anti)symmetry under exchange of particles 2 and 3. Therefore the orbital angular momentum part of the wave function is the same as for non-identical spin-zero particles 2 and 3. Furthermore, due to separability for $m_{1}=\infty$ and $V_{23}=0$, the three-body states with different orbital angular momentum $L$ are degenerate even if particles 2 and 3 are identical.

The only exception is when particles 2 and 3 are identical bosons with zero spin. Then the spin part in the wave function is not available to establish the symmetry under exchange of the particles, and the odd values of the relative orbital angular momentum between the two bosons are therefore strictly forbidden. This has consequences even when $V_{23}=0$. For instance, if the core-boson interaction only has a $p$-wave, the minimum value of the hypermomentum for the even parity states should normally be $K_{\min }=2$. However, to get $L=1$, the smallest values of the relative orbital angular momenta are 2 both between the bosons and between their center of mass and the core. This implies that $K_{\min }=4$. The effective radial potentials therefore vary strongly from $L=1$ with $K_{\min }=4$ to $L=0,2$ where $K_{\min }=2$, see [1].

We need a reliable method to compute three-body resonances and bound states. We choose the Faddeev hyperspherical adiabatic expansion method $[1,6]$ combined with the complex scaling method $[5,7,8]$. For this we define the only length coordinate, the hyperradius $\rho$, as

$$
m \rho^{2} \equiv \frac{1}{M} \sum_{i<k} m_{i} m_{k}\left(\boldsymbol{r}_{i}-\boldsymbol{r}_{k}\right)^{2},
$$


where $\boldsymbol{r}_{i}$ is the coordinate of particle $i, M=\sum m_{i}$ and $m$ is an arbitrary normalization mass. The complex scaling then amounts to the substitution $\rho \rightarrow$ $\rho \exp (i \theta)$ in the Faddeev equations. The wave function of a resonance then falls off exponentially when the angle $\theta$ is larger than $\theta_{r} \equiv \arctan \left(\kappa_{I} / \kappa_{R}\right)$, i.e., the angle corresponding to the complex momentum $\kappa=\kappa_{R}-i \kappa_{I}=\sqrt{2 m E / \hbar^{2}}$ of the complex resonance energy $E$. The asymptotic large $\rho$ behavior of the different radial wave functions $f_{n n^{\prime}}$ are [1,5]

$f_{n n^{\prime}} \rightarrow \sqrt{\rho} H_{K+2}^{(1)}\left(|\kappa| \rho e^{i\left(\theta-\theta_{R}\right)}\right)$,

where $K$ is the integer (hypermomentum) related to the asymptotic adiabatic radial potential and $H_{K+2}^{(1)}$ is the outgoing Hankel function of first kind. Bound states with the true real and negative energies are also eigensolutions to the complex rotated equations.

\section{P-waves: ${ }^{6} \mathrm{He}$}

Let us consider an infinitely heavy core $\left(m_{1}=\infty\right)$ and two non-interacting identical particles 2 and 3
$\left(V_{23}=0\right)$ such that $\mu_{12}=\mu_{13}=0.8 m$, where $m$ is the nucleon mass. The core-particle interaction is a spin-independent Gaussian acting only on $p$ waves with a two-body resonance energy and width $\left(E_{R}, \Gamma_{R}\right)=(0.74,0.61) \mathrm{MeV}$ indicated by the cross in Fig. 1. These parameters correspond to the lowest $p_{3 / 2}$-resonance in ${ }^{5} \mathrm{He}$. The three-body system has a resonance with twice the energy and width, i.e., $\left(E_{R}, \Gamma\right)=(1.48,1.22) \mathrm{MeV}$ indicated by a big open circle in Fig. 1. The degeneracy is 3 corresponding to the orbital angular momenta $L^{\pi}=0^{+}, 1^{+}, 2^{+}$except for two identical bosons with spin zero where $L^{\pi}=1^{+}$is excluded for symmetry reasons.

In Fig. 1 we show how the resonances of $L^{\pi}=0^{+}$, $1^{+}$, and $2^{+}$vary as $m_{1}$ changes from $\infty$ to $m$ (solid symbols). The degeneracy is broken and all three states move towards more binding resulting entirely from the changing contribution of the center of mass kinetic energy. The $L=1$ three-body state is even bound for $m_{1}=m$. Thus a Borromean state can exist even if two of the particles do not interact.

Along these three curves the points indicated by the thick arrows in Fig. 1 correspond to $m_{1}=4 m$ and $m_{2}=m_{3}=m$. This three-body system is simi-

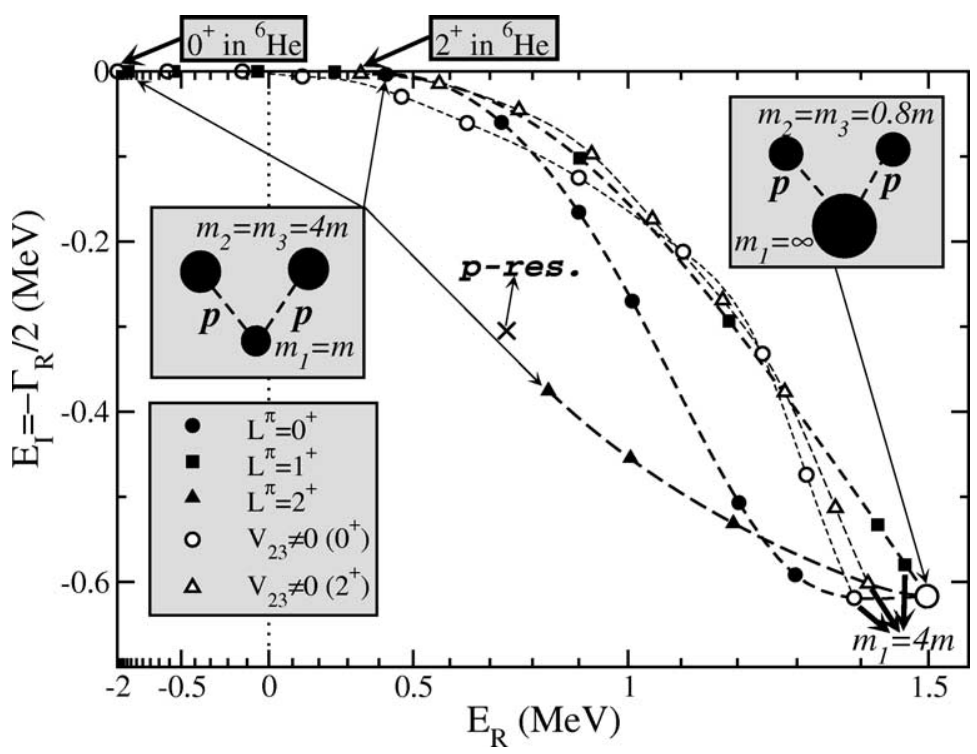

Fig. 1. Real and imaginary values of resonance energies for a three-body system with reduced masses $\mu_{12}=\mu_{13}=0.8 \mathrm{~m}$ ( $\mathrm{m}$ is the nucleon mass). The cross indicates the position of the $p$-resonance for the 1-2 and 1-3 two-body systems. The big open circle indicates the three-body resonance for $m_{1}=\infty$ and $V_{23}=0$. The solid circles, solid squares, and solid triangles show the energies of the $0^{+}, 1^{+}$, and $2^{+}$resonances as function of $m_{1}$. The open circles and open triangles show the energies of the $0^{+}$and $2^{+}$resonances for $m_{1}=4 m$ when $V_{23}$ change from zero to full neutron-neutron strength. 
lar to ${ }^{6} \mathrm{He}$, except that the interaction $V_{23}$ is equal to zero. We now introduce the realistic spin-dependent neutron-neutron interaction $V_{23}$ multiplied by a factor varying from 0 to 1 . The $0^{+}$and $2^{+}$states then move from the close-lying $m_{1}=4 m$ points to respectively binding of $2 \mathrm{MeV}$ (Borromean state) and the resonance position $\left(E_{R}, \Gamma_{R}\right)=(0.34 \mathrm{MeV}, 0.01 \mathrm{MeV})$. The effect of changing from $m_{1}=\infty$ to $m_{1}=4 m$ is small, and therefore the effect responsible for the existence of the $0^{+}$Borromean state and the lowlying $2^{+}$resonance is the interaction between the neutrons.

The computed $0^{+}$and $2^{+}$states in the schematic model are more bound than the experimental values for the corresponding states in ${ }^{6} \mathrm{He}$. This overbinding has two main sources. First, the core-neutron spinorbit interaction is neglected and the $p_{1 / 2}$ resonance appears at $0.74 \mathrm{MeV}$ instead of the $2 \mathrm{MeV}$ measured for ${ }^{5} \mathrm{He}$. Second, we neglected the highly repulsive alpha-neutron $s$-wave interaction which accounts for the Pauli principle by excluding overlap to the neutron $s$-state within the alpha particle. A detailed calculation taking these facts into account is available in $[9,10]$.

The neutron-neutron interaction is predominantly of $s$-wave character, and an $L=1$ state is therefore only possible with a relative $p$-state between core and center of mass of the two neutrons. Then the neutronneutron interaction has no effect on the $1^{+}$-state which remains at the initial $m_{1}=4 m$ point. Furthermore, the $1^{-}$state does not appear numerically as a resonance, because one virtual $s$-state and one $p$-resonance produce an $S$-matrix pole on the unphysical sheet. We have not found any $1^{-}$resonance even for a realistic alpha-neutron interaction with a repulsive partial $s$-wave.

\section{Mixed S- and P-waves: ${ }^{11} \mathrm{Li}$}

We now extend to include both $s$ and $p$-waves. Particles 2 and 3 are again identical particles, the interactions $V_{12}=V_{13}$ with reduced masses $\mu_{12}=$ $\mu_{13}=0.9 m$ produce a virtual $s$-state at $-0.54 \mathrm{MeV}$ and a $p$-resonance with $\left(E_{R}, \Gamma_{R}\right)=(1.38,2.77) \mathrm{MeV}$ (crosses in Fig. 2). For $V_{23}=0$ the computations in practice correspond to $m_{1}=9 m, m_{2}=m_{3}=m$ which for $p$-waves alone leads to resonances similar to the results for $m_{1}=\infty$, see Fig. 1 . As in the previous section, for pure $p$-waves, the $L^{\pi}=0^{+}, 1^{+}, 2^{+}$threebody resonances are given by twice the energy of

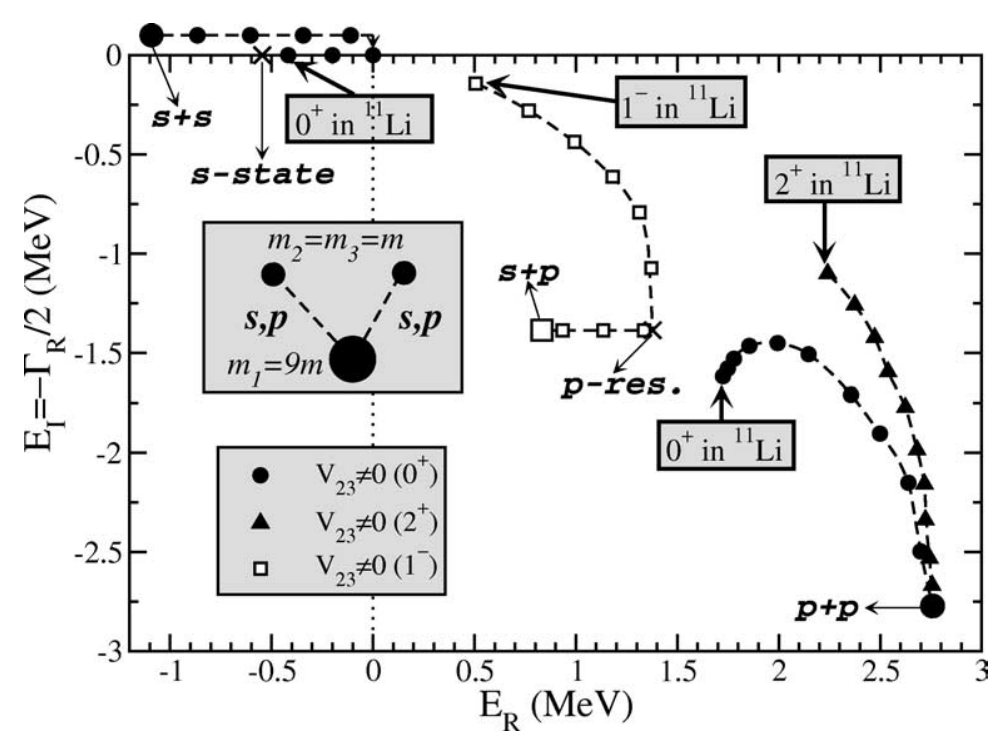

Fig. 2. Real and imaginary values of resonance energies for a three-body system with reduced masses $\mu_{12}=\mu_{13}=0.9 m$ ( $m$ is the nucleon mass). The crosses indicate the positions of virtual $s$-state and $p$-resonance for the 1-2 and 1-3 two-body systems. The big solid circles and big open square indicate the three-body poles for $V_{23}=0$. The solid circles, solid triangles, and open squares show the energies of the $0^{+}, 2^{+}$, and $1^{-}$states for $m_{1}=9 m$ when $V_{23}$ becomes finite. 
the two-body $p$-resonance (big solid circle labeled as $p+p$ in Fig. 2).

In addition there is a second $0^{+}$virtual state with only $s$-wave components, and a $1^{-}$pole with mixed two-body $s$ and $p$-components. Both these $S$-matrix poles appear on the unphysical Riemann sheet at twice the two-body $s$-state energy and at the sum of $s$ and $p$ energies, respectively (big solid circle labeled as $s+s$ and big open square in Fig. 2). They cannot be found numerically with the complex scaling method [10], but a sufficiently strong attractive interaction $V_{23}$ moves the pole onto the physical sheet where they appear as resonances [5].

We choose again $V_{23}$ as the realistic neutronneutron interaction multiplied by a factor varying from 0 to 1 . The two identical fermions with spin $1 / 2$ suppress the $1^{+}$. The evolution with the strength of $V_{23}$ is shown in Fig. 2. The $0^{+}$and the $2^{+}$states, originating exclusively from the $p$-components, both move towards more binding arriving at $\left(E_{R}, \Gamma_{R}\right)=$ $(1.73,3.24) \mathrm{MeV}$ and $(2.24,2.20) \mathrm{MeV}$ for the $0^{+}$ and $2^{+}$states, respectively. For full neutron-neutron strength the core-neutron $p$-wave components in the $0^{+}$state correspond to a probability of about $80 \%$ whereas the $2^{+}$state entirely must be $100 \% p$-waves. The $2^{+}$state has a smaller width and a larger energy than the $0^{+}$state, but they are both far from being bound states.

The other $0^{+}$state, originating exclusively from two-body $s$-components, is initially a virtual threebody pole on the negative energy axis of the unphysical Riemann sheet. As the attraction $V_{23}$ is switched on this pole moves towards the origin and continues smoothly onto the physical sheet and becomes a Borromean bound state of energy $E_{R}=-0.42 \mathrm{MeV}$. The core-neutron $p$-wave components in this state is slightly above a probability of $30 \%$.

The $1^{-}$state originating from mixed $s$ and $p$ twobody states is also initially on the unphysical sheet, but as $V_{23}$ is continuously switched on the pole appears at some point as a resonance with the complex energy of the two-body $p$-resonance [5]. This evolution is shown in Fig. 2 with the arrival for full neutronneutron interaction at $\left(E_{R}, \Gamma_{R}\right)=(0.51,0.29) \mathrm{MeV}$. The core-neutron components remain for parity reasons a mixture of $s$ - and $p$-states.

The spectrum obtained with the full neutronneutron interaction is the one of ${ }^{11} \mathrm{Li}$ with the assump- tion of zero core spin. It is known experimentally that ${ }^{11} \mathrm{Li}$ has a Borromean $0^{+}$bound state at $-0.3 \mathrm{MeV}$ with a $p$-wave content of $40 \%-50 \%$, and also evidences for a $1^{-}$resonance at about $1 \mathrm{MeV}$ [11] have been found. There is not experimental information about other resonances, but different calculations have predicted additional excited states [12].

The ${ }^{11} \mathrm{Li}$ calculations shown in Fig. 2 have unrealistic features. The core-neutron interaction should be more attractive to reproduce the experimental constraints of an $s$-state below $100 \mathrm{keV}$ and a $p$-resonance at around $0.5 \mathrm{MeV}$. The overbinding mentioned in connection with ${ }^{6} \mathrm{He}$ is also a problem here due to neglect of spin-orbit potential and the simplified treatment of the Pauli principle. However, a conceptually different problem is the spin dependence of the core-neutron interaction due to the finite spin of the ${ }^{11} \mathrm{Li}$ core. The consequence is an unavoidable spin-splitting lifting the degeneracy from the beginning and furthermore lowering the crucial two-body virtual state and resonance energies. All these effects, partially canceling each other, conspire to make the ${ }^{11} \mathrm{Li}$ system complicated.

Still the interactions used in Fig. 2 provide a realistic illustration of the relative sizes of the principal effects. In particular the ground state originates from the combination of two $s$-components, and the first rather low-lying $1^{-}$excited state originates from the mixing of $s p$-components. Also, additional excited states $\left(0^{+}\right.$and $\left.2^{+}\right)$arise from the two $p$-components. In fact these four states are consistent with the spectrum found for a system like ${ }^{12} \mathrm{Be}$, that it is known experimentally to have a bound $0^{+}$state, a $0^{+}$resonance, a $1^{-}$resonance, and a $2^{+}$resonance $[13,14]$.

\section{Realistic ${ }^{11} \mathrm{Li}$ : finite core spin}

The immediate consequence of a non-zero core spin $s_{1}$ is the increase in the number of low-lying core-neutron resonances. Every core-neutron level of angular momentum $j_{2}$ is split into a series of states with total angular momentum from $\left|j_{2}-s_{1}\right|$ to $j_{2}+s_{1}$. A realistic model should then reproduce experimental information of (the most important of) the degeneracies and complex energies of these states. Then the neutron-core interaction necessarily contains spinspin and spin-orbit operators which must be chosen 


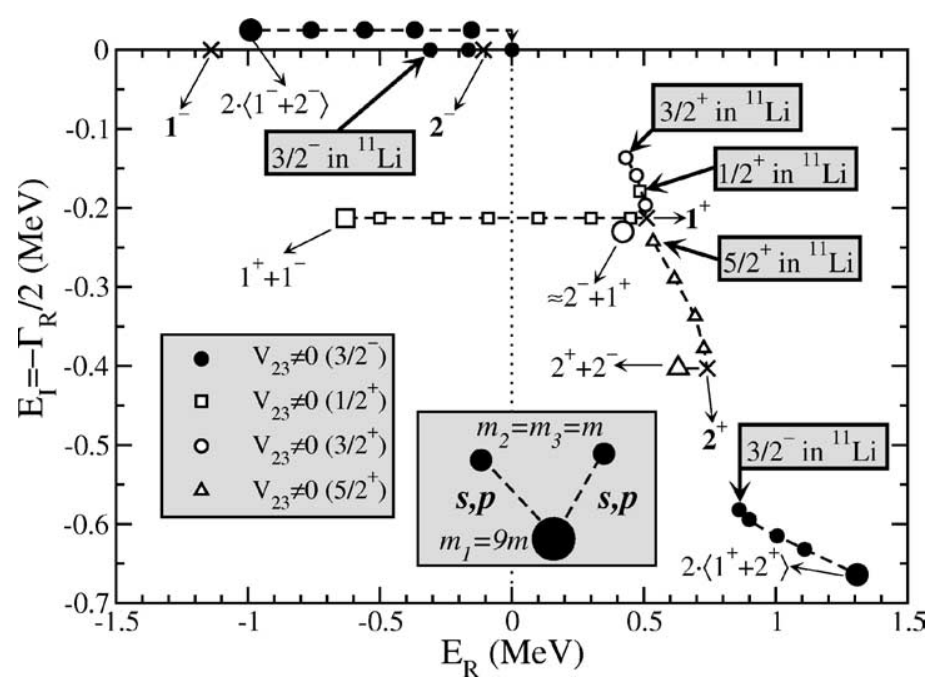

Fig. 3. Real and imaginary values of resonance energies for ${ }^{11} \mathrm{Li}$ with finite core spin of $3 / 2$. The reduced masses are $\mu_{12}=\mu_{13}=0.9 m$. The two-body systems 1-2 and 1-3 have virtual $s$-states and $p$-resonances at the energies indicated by the crosses. The big symbols are the three-body states of the system for $V_{23}=0$. The solid circles, open squares, open circles, and open triangles show the energies of the $3 / 2^{-}$, $1 / 2^{+}, 3 / 2^{+}$, and $5 / 2^{+}$states when the neutron-neutron interaction is progressively increased.

judiciously. This is particularly important when the particles surrounding the core are fermions identical to those within the core. Consistency between the antisymmetrization and the spin operators is indispensable to avoid catastrophic results for the three-body system [15].

To trace the origin of such Borromean systems exemplified by ${ }^{11} \mathrm{Li}$ with core-spin and parity $3 / 2^{-}$we again turn to the schematic model where $m_{1}=\infty$ and $V_{23}=0$. The three-body Hamiltonian $H$ is still a sum of two two-body Hamiltonians $H_{12}$ and $H_{13}$ with eigenvalues $E_{12}^{\left(j_{12}\right)}$ and $E_{13}^{\left(j_{13}\right)}$, respectively. The states are labeled by the two-body angular momenta $j_{12}$ and $j_{13}$ obtained by coupling the spin $s_{1}$ of the core and the total two-body angular momenta $j_{2}$ and $j_{3}$ of the particles in their motion relative to the core.

The relevant ${ }^{9} \mathrm{Li}$-neutron states are the $s_{1 / 2}$ and $p_{1 / 2}$ partial waves which, combined with spin and parity of the core, result in the two ${ }^{10} \mathrm{Li}$ doublets $1^{-} / 2^{-}$and $1^{+} / 2^{+}$. We choose these ${ }^{10} \mathrm{Li}$ continuum states at $-0.10 \mathrm{MeV}\left(2^{-}\right),-1.14 \mathrm{MeV}\left(1^{-}\right)$, $0.51-i 0.21 \mathrm{MeV}\left(1^{+}\right)$, and $0.74--i 0.40 \mathrm{MeV}\left(2^{+}\right)$. These energies (crosses in Fig. 3) are slightly above the ones suggested by the experiments and by more sophisticated calculations [16]. This allows us to reproduce the experimental three-body energy with the present model.
When only the $s_{1 / 2}$ and $p_{1 / 2}$ waves are considered in ${ }^{10} \mathrm{Li}$, the possible three-body ${ }^{11} \mathrm{Li}$ states are characterized by total angular momentum and parity $j^{\pi}=1 / 2^{ \pm}, 3 / 2^{ \pm}, 5 / 2^{ \pm}$. The ground state has $j^{\pi}=$ $3 / 2^{-}$and the positive parity states correspond to the $1^{-}$-excitations. These three-body states are antisymmetric under neutron interchange. In analogy to the measured structure of the ${ }^{12} \mathrm{Be}$ spectrum [14] the $s_{1 / 2}$ and $p_{1 / 2}$ states inevitably lead to two $3 / 2^{-}$states.

In the schematic model the energies of the two $\left(j^{\pi}=3 / 2^{-}\right)$-states are twice the average energy of the doublets corresponding to two neutrons either in the $1^{-} / 2^{-}\left(100 \% s^{2}\right)$ or in the $1^{+} / 2^{+}\left(100 \% p^{2}\right)$ states. These average energies are shown in Fig. 3 as big solid circles at $-0.99 \mathrm{MeV}$ and $1.31-i 0.66 \mathrm{MeV}$. The correct finite core mass only moves these points very little. When the neutron-neutron interaction is progressively switched on the first $3 / 2^{-}$-state moves along the negative energy axis until the system becomes bound and at full strength the Borromean state is found at $-0.30 \mathrm{MeV}$ with the $s^{2}$ content reduced to $70 \%$. The second $3 / 2^{-}$-state also moves and reaches for full strength the resonance position $\left(E_{R}, \Gamma_{R}\right)=(0.86,1.16) \mathrm{MeV}$ with the $p^{2}$ content reduced to $93 \%$.

The structure of the $1^{-}$-excitations must correspond to one neutron in one of the two $s$-states in ${ }^{10} \mathrm{Li}$, 
and the other neutron in one of the $p$-states. For the $1 / 2^{+}$and the $5 / 2^{+}$states, the two neutrons necessarily occupy the $1^{-} / 1^{+}$and $2^{-} / 2^{+}$states, respectively. Therefore, in the schematic model the three-body energies are $E_{12}^{(1)}+E_{13}^{(1)}$ and $E_{12}^{(2)}+E_{13}^{(2)}$ as shown in Fig. 3 by a big open square and a big open triangle. These states are not three-body resonances, but virtual $s$-states with respect to the two-body resonance. When the neutron-neutron interaction increases, at some point these poles on the unphysical Riemann sheet appear as three-body resonances through the two-body resonance energies indicated by the small open squares and the small open triangles.

Finally, in the schematic model two $3 / 2^{+}$-states are found at $0.42-i 0.23 \mathrm{MeV}$ and $-0.42-i 0.38 \mathrm{MeV}$, which approximately correspond to the neutrons in the $2^{-} / 1^{+}$and the $1^{-} / 2^{+}$-states, respectively. Only the first of these states shows up as a three-body resonance when the neutron-neutron interaction is switched on. The other pole remains on the unphysical Riemann sheet. All the three $1^{-}$resonances move towards the origin reaching an energy of about $0.5 \mathrm{MeV}$ and widths of $0.3-0.5 \mathrm{MeV}$. Evidence for a combination of these overlapping resonances is available [11].

The degeneracy of these $1^{-}$-excitations is broken by the core-spin dependence of the ${ }^{9} \mathrm{Li}$-neutron interaction. The resonance positions are very sensitive to this spin dependence which must be chosen consistent with the Pauli principle and the mean-field treatment of the neutrons within the core. Violation of these constraints can rather easily lead to very wrong energies of the $1^{-}$-resonances or even to bound states.

\section{Conclusions}

We have traced the origin of Borromean systems to the properties of the two-body subsystems. One infinitely heavy core mass and two non-interacting light particles is a separable system where the complex energies of the $S$-matrix poles are obtained by adding the corresponding two-body energies. The three-body states then lie higher than the individual two-body states. The existence of Borromean bound states means that attractive effects must overcompensate for this initial loss of energy in the three-body system. Three contributions are responsible, i.e., the finite core mass reduces the positive kinetic energy, the angular momentum couplings favor special structures, and the light particle interactions are attractive in specific partial waves.

We illustrate with established Borromean nuclei where two neutrons surround the core. Then only the short-range interaction contributes. Inclusion of the Coulomb repulsion destroys the initial assumption of separability for an infinitely heavy core. The poles would move towards less binding and the widths would be much smaller due to the repulsive and confining effects of the Coulomb barrier. Otherwise we expect the same qualitative behavior.

We conclude that Borromean systems can arise separately from each of the above three contributions. With known energies of low-lying virtual states and resonances we can predict both the relative importance of the three effects and the properties of the resulting three-body ground and excited states. These investigations have only recently become possible with the implementation of the powerful techniques to handle both discrete and continuum three-body states.

\section{References}

[1] E. Nielsen, D.V. Fedorov, A.S. Jensen, E. Garrido, Phys. Rep. 347 (2001) 373.

[2] A. Ozawa, T. Suzuki, I. Tanihata, Nucl. Phys. A 693 (2001) 32.

[3] P.G. Hansen, A.S. Jensen, B. Jonson, Annu. Rev. Nucl. Part. Sci. 45 (1995) 591.

[4] A.S. Jensen, K. Riisager, D.V. Fedorov, E. Garrido, Rev. Mod. Phys. 76 (2004) 215.

[5] E. Garrido, D.V. Fedorov, A.S. Jensen, Few Body Systems 34 (2004) 91.

[6] A.S. Jensen, E. Garrido, D.V. Fedorov, Few Body Systems 22 (1997) 193.

[7] Y.K. Ho, Phys. Rep. 99 (1983) 1.

[8] T. Myo, K. Kato, S. Aoyama, K. Ikeda, Phys. Rev. C 63 (2001) 054313.

[9] E. Garrido, D.V. Fedorov, A.S. Jensen, Nucl. Phys. A 617 (1997) 153.

[10] D.V. Fedorov, E. Garrido, A.S. Jensen, Few Body Systems 33 (2003) 153

[11] A.A. Korsheninnikov, et al., Phys. Rev. C 53 (1996) R537.

[12] S. Aoyama, K. Kato, T. Myo, K. Ikeda, Prog. Theor. Phys. 107 (2002) 543.

[13] H. Iwasaki, et al., Phys. Lett. B 491 (2000) 8.

[14] S. Shimoura, et al., Phys. Lett. B 560 (2003) 31.

[15] E. Garrido, D.V. Fedorov, A.S. Jensen, Phys. Rev. C 68 (2003) 014002 .

[16] E. Garrido, D.V. Fedorov, A.S. Jensen, Nucl. Phys. A 700 (2002) 117 